CHEMOPREVENTION

\section{Acting on active AKT}

Improving early detection, decreasing tobacco smoking and developing better therapies are all important strategies for combatting lung cancer, but drug-targeted lung cancer prevention is another option. Ho-Young Lee and colleagues now show that AKT - a downstream component of the phosphatidylinositol 3-kinase (PI3K) pathway, which is important in regulating cell proliferation and apoptosis - is constitutively active in an in vitro lung carcinogenesis progression model. They further report that deguelin - a natural plant product drug — inhibits activated AKT in this model system, so showing potential as a lung cancer chemoprevention agent.

Ho-Young Lee and colleagues used normal, immortalized, premalignant and malignant human bronchial epithelial (HBE) cell lines. Deguelin inhibited cell proliferation - cells accumulated in the G2-M phase of the cell cycle - and induced apoptosis in the premalignant and malignant cell lines in a dose- and time-dependent manner, but had no adverse effects on normal or immortalized HBE cells. Levels of activated phosphorylated AKT were higher in the transformed cells than in normal cells and, at concentrations that might be attainable in vivo, the authors showed that deguelin decreased levels of phosphorylated AKT without affecting the total levels of the protein. Deguelin also decreased PI3K activity by about half, although it took longer to do so than inhibition of AKT, indicating that deguelin might inhibit AKT activity through both PI3K-dependent and -independent mechanisms.

So, is the AKT pathway a specific target of deguelin? Treatment with deguelin did not affect the activity of other components of kinase pathways, such as mitogen-activated protein kinase (MAPK), extracellular signal-related kinase 1/2 (ERK1/2) or JUN N-terminal kinase (JNK). In addition, when one of the premalignant cell lines was infected with an adenovirus expressing constitutively

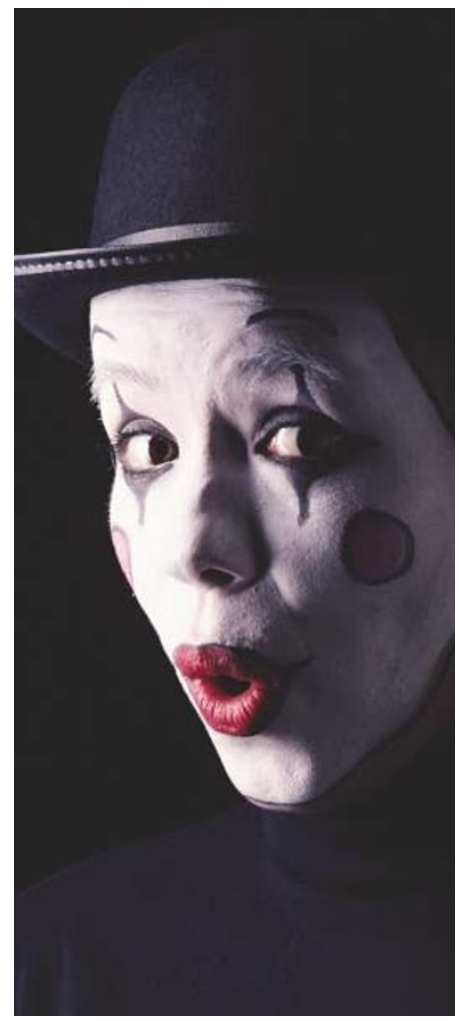

active AKT and treated with deguelin, inhibition of growth and triggering of apoptosis was much reduced.

As deguelin belongs to a class of agents that are used as insecticides, which have been associated with cardiac and other toxicities in humans, thorough evaluation of possible toxic effects will be key to the development of the compound for lung cancer prevention. Constitutive activation of AKT occurs frequently in non-smallcell lung cancer in humans, so we await further investigation of how deguelin inhibits AKT with interest.

Ezzie Hutchinson

\section{6) References and links} ORIGINAL RESEARCH PAPER Chun, K.-H. et al. Effects of deguelin on the

phosphatidylinositol 3-kinase/Akt pathway and apoptosis in premalignant human bronchial epithelial cells. J. Natl Cancer Instit. 95, 291-301 (2003)

FURTHER READING Vivanco, I. \& Sawyers, C. L. The phosphatidylinositol 3-kinase-AKT pathway in human cancer. Nature Rev. Cancer. 2, 489-501 (2003)

\section{WEB SITE}

Waun Ki Hong's lab:

http://www.mdanderson.org/departments/

thoracic_hnmo/

\section{TRIAL WATCH}

\section{An aspirin a day...}

A daily dose of aspirin can reduce the incidence of colorectal polyps in people at high risk for colorectal cancer, according to two randomized controlled clinical trials published in the 6 March issue of New England Journal of Medicine.

Sandler et al. conducted a randomized, double-blind trial of 635 patients with previous colorectal cancer. Patients were given either $325 \mathrm{mg}$ per day of coated aspirin or placebo. One or more adenomas developed in $27 \%$ of patients in the placebo group, but only in $17 \%$ of patients in the aspirin group. The trial was therefore terminated after only 31 months, to allow the placebo group to go onto aspirin therapy. The mean size of the adenomas, however, did not differ significantly between groups, nor did the percentage of patients with advanced adenomas. The hazard ratio for new polyp formation in the aspirin group, compared with the placebo group, was 0.64 , indicating that aspirin delayed the development of adenomas. Adverse effects were infrequent. Baron et al. studied 1,121 patients who had also recently had adenomas removed. These patients were randomly assigned to receive either placebo, $81 \mathrm{mg}$ or $325 \mathrm{mg}$ of aspirin per day. After 33 months, $12.9 \%$ of the patients in the placebo group developed advanced neoplasms, compared with $7.7 \%$ of those in the $81 \mathrm{mg}$ group and $10.7 \%$ of those in the $325 \mathrm{mg}$ group. The reason that the $81-\mathrm{mg}$ dose was more beneficial than the $325-\mathrm{mg}$ dose is unclear, and requires further study. Stroke and serious bleeding were more frequent in the patients that took aspirin, but the differences were not statistically significant.

These data confirm earlier observational studies showing that aspirin - a non-steroidal anti-inflammatory drug that downregulates cyclooxygenase (COX) enzymes - can reduce the incidence of colorectal adenomas. Several epidemiological studies have shown an association between COX inhibitors and a reduced risk of colorectal neoplasia - despite differences in study designs. In randomized trials involving patients with familial polyposis, sulindac and celecoxib were shown to reduce the number and size of adenomatous polyps. However, these are the first randomized trials to show the effects of aspirin in preventing adenoma recurrence in patients with sporadic colorectal cancer.

In an accompanying editorial, Thomas F. Imperiale (Indiana University School of Medicine) states "Although aspirin may be of some benefit in preventing colorectal cancer, it cannot yet be recommended for this indication and is not a substitute for screening and surveillance". Nevertheless, these findings show that aspirin moderately reduces the risk of recurrent colorectal neoplasia. Future clinical trials will determine whether aspirin can be used to decrease the frequency or intensity of screening or surveillance that is current practice.

ORIGINAL RESEARCH PAPERS Sandler, R. S. et al. A randomized trial of aspirin to prevent colorectal adenomas in patients with previous colorectal cancer. N. Engl. J. Med. 348, 883-890 (2003) | Baron, J. A. et al. A randomized trial of aspirin to prevent colorectal adenomas. N. Engl. J. Med. 348, 891-899 (2003)

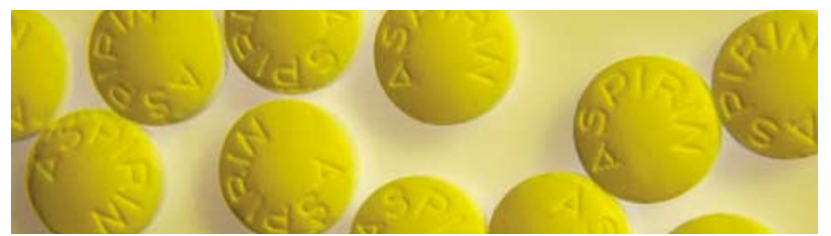

